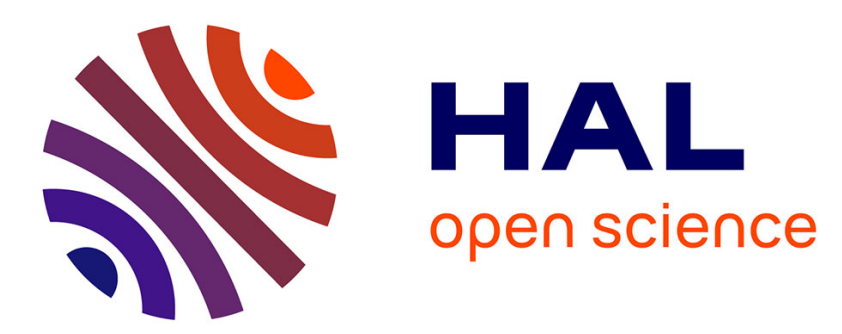

\title{
Influence of a Full-Body Compression Suit on Trunk Positioning and Knee Joint Mechanics During Lateral Movements
}

Guillaume Mornieux, Elmar Weltin, Monika Pauls, Franz Rott, Albert Gollhofer

\section{To cite this version:}

Guillaume Mornieux, Elmar Weltin, Monika Pauls, Franz Rott, Albert Gollhofer. Influence of a FullBody Compression Suit on Trunk Positioning and Knee Joint Mechanics During Lateral Movements. Journal of Applied Biomechanics, 2017, 33 (4), pp.261-267. 10.1123/jab.2016-0061 . hal-03102210

\section{HAL Id: hal-03102210 \\ https://hal.univ-lorraine.fr/hal-03102210}

Submitted on 23 Apr 2021

HAL is a multi-disciplinary open access archive for the deposit and dissemination of scientific research documents, whether they are published or not. The documents may come from teaching and research institutions in France or abroad, or from public or private research centers.
L'archive ouverte pluridisciplinaire HAL, est destinée au dépôt et à la diffusion de documents scientifiques de niveau recherche, publiés ou non, émanant des établissements d'enseignement et de recherche français ou étrangers, des laboratoires publics ou privés. 


\section{December $\mathbf{2 9}^{\text {th }}, \mathbf{2 0 1 6}$}

2 JAB_2016_0061.R3

3

$4 \quad$ Influence of a full body compression suit on trunk positioning and knee joint mechanics during lateral movements.

6

$7 \quad$ Mornieux Guillaume ${ }^{1}$, Weltin Elmar ${ }^{2}$, Pauls Monika $^{3}$, Rott Franz ${ }^{4}$, and Gollhofer Albert ${ }^{2}$

$9 \quad{ }^{1}$ Faculty of Sport Sciences, EA $3450-$ DevAH, University of Lorraine, Nancy, France;

${ }^{2}$ Institute of Sport and Sport Science, University of Freiburg, Freiburg, Germany; ${ }^{3}$ Institute of Sport Science, Technische Universität Kaiserslautern, Kaiserslautern, Germany; ${ }^{4}$ adidas AG, Herzogenaurach, Germany

14 Funding: this work was supported by the adidas AG via a research grant.

15 Conflict of Interest Disclosure: the authors have no conflict of interest to declare.

16 Correspondence Address: Guillaume Mornieux

17 University of Lorraine - Faculty of Sport Sciences - EA $3450-$ DevAH

1830 rue du Jardin Botanique - 54600 Villers-lès-Nancy, France

19 Tel.: +33(0)383682931/ Fax: +33(0)3 83682902

20 Email: guillaume.mornieux@univ-lorraine.fr

21 Running Title: Full body compression suit and lateral movements. 
Trunk positioning has been shown to be associated with knee joint loading during athletic tasks, especially changes of direction. The purpose of the present study was to test whether a full body compression suit (FBCS) would improve trunk positioning and knee joint control during lateral movements. Twelve female athletes performed lateral reactive jumps (LRJ) and unanticipated cuttings with and without the customized FBCS, while 3D kinematics and kinetics were measured. FBCS did not influence trunk positioning during LRJ and led to increased trunk lateral lean during cuttings $(P<.001)$. However, while wearing FBCS, knee joint abduction and internal rotation angles were reduced during LRJ $(P<.001$ and $P=.013$, respectively), whereas knee joint moments were comparable during cuttings. FBCS cannot support the trunk segment during unanticipated dynamic movements. But increased trunk lateral lean during cutting maneuvers was not high enough to elicit increased knee joint moments. On the contrary, knee joint abduction and internal rotation were reduced during LRJ, speaking for a better knee a full body compression suit.

38

Keywords: cuttings, perturbation, trunk lateral lean, knee abduction moment, agility.

Word Count: 3995 words 
Lateral movements with changes of direction are common in many team sports such as soccer or handball. During cutting maneuvers, the athlete performs a complex dynamic task by quickly changing their direction of movement while securing their balance. Knee joint control is an essential requirement during such movements to prevent misalignments in the lower extremities with the consequence that the knee may be injured. ${ }^{1,2}$ Non-contact anterior cruciate ligament (ACL) injury mechanism during cutting maneuvers typically involves knee external flexion moment combined with dynamic valgus rotation with the body weight shifted over the injured leg. ${ }^{2}$ Trunk positioning during lateral movements is of interest as it would influence the location of the body weight, and potential knee joint loads. ${ }^{3,4}$ Hewett and Myer ${ }^{3}$ proposed a knee injury model that accounted for both the trunk and hip joints, because increased trunk lateral lean is reportedly associated with larger external knee abduction moments. Greater lateral trunk position has been shown during anterior cruciate ligament injury situations in female athletes. ${ }^{5}$ Different laboratory studies using cutting maneuvers and lateral reactive jumps paradigms have demonstrated that increased trunk lateral lean is positively associated with increased knee abduction moments. ${ }^{6-8}$ Further, increased knee joint loading has been associated with increased trunk rotation away from the stance limb. ${ }^{8,9}$ For instance, higher knee internal rotation moment was found for trunk rotation in the opposite direction to the cutting maneuver. ${ }^{8}$ Accordingly, any improvement of trunk positioning is of interest with regard to knee joint loading. This would be especially true for female athletes, whose incidence of ACL injuries in sports such as soccer and basketball is much higher than male athletes. ${ }^{10,11}$ In sport sciences, the influence of compression garments, providing passive support to different body segments during exercise has been extensively studied. ${ }^{12}$ It has been showed that compressive and elastic garments could reduce the range of motion of the joint. ${ }^{13,14}$ Compression shorts reduced the active range of motion during hip flexion in a non-athletic 
$\operatorname{task}^{13}$ and hip range of motion during a $60 \mathrm{~m}$ sprint. ${ }^{14}$ Wearing shorts with directional compression was associated with reduced hip adductor activity during cutting manoeuvres. ${ }^{15}$ In this latter study, the use of diagonal bands of fabric that only stretch along their length increased the compression level along the line of action of hip adductors. Therefore, apparel, through their compression and elasticity properties, could be used to passively support body segments and joints during various tasks. A compression suit that would functionally support the trunk over the pelvis and leg could be a solution to avoid trunk positioning in the opposite direction to the intended movement. Such apparel would work as external trunk stabilizers to improve trunk positioning during various tasks, especially lateral movements, and subsequently possibly reduce knee joint loading.

In sports, any improvement of movement control would further be relevant if associated with enhanced performance. However, when considering whole-body dynamic tasks, wearing a compression suit did not influence performance in cricket players ${ }^{16}$ or endurance performance during running. ${ }^{17}$ Only one study has shown possible benefits of a whole-body compression garment on performance during high intensity intermittent exercises, mainly because of better muscle oxygenation. ${ }^{18}$ Therefore, athletic performance is not likely to be increased when using garments.

The aim of this present study was to test whether compression garments can improve trunk and hip positioning and resultant knee joint loading, while not decreasing performance during dynamic lateral sporting movements. For that purpose, a full body compression suit with functional elastic straps was designed to improve trunk positioning and knee joint control during lateral movements. We tested the hypotheses that i) trunk and hip joint angles would be reduced with the compression suit, ii) knee joint abduction moment would be reduced with the compression suit and iii) exercise performance level would be maintained while wearing the compression suit. 
According to other studies evaluating comparable dependent measures during unanticipated lateral movements ${ }^{19}$ or the influence of compression shorts on sports performance, ${ }^{13}$ a sample size of 13 participants was estimated relevant for statistical analysis. The rationale for focusing on females was their relative higher risk of sustaining non-contact ACL injuries. ${ }^{10}$ Among the 13 female athletes recruited to participate to the study, one was injured during a training session with her team during the investigation period and could not take part in the measurements. Therefore, 12 female athletes (body height $=171 \pm 4 \mathrm{~cm}$; body mass $=59 \pm 4 \mathrm{~kg}$; age $=22 \pm 2$ years) who were familiar with lateral movements (soccer, basketball and handball) completed the entire study. Participants did not have a previous history of serious knee injury or any current knee pain. Written consent was obtained prior to study participation. The study was approved by the local ethics committee (approval 72/14) and was 105 conformed to requirements stipulated in the Declaration of Helsinki.

During a first visit, participants' body dimensions were scanned (BodyKinectizer, adidas IT Innovation, Herzogenaurach, Germany), in order to customize the full body compression suit. During a second visit at our laboratory, participants performed different lateral movements and performance tests under two different apparel conditions, either while wearing the customized full body compression suit (FBCS) with 2x thermoplastic polyurethane

111 (TPU) layers or with standard sport shorts and bras (Control). FBCS was a prototype specially designed for the present study in order to provide support for the trunk over the pelvis segment 113 in three dimensions. For that purpose, a mean compression level of $5.2 \mathrm{mmHg}$ was achieved 114 and the 2x TPU layers design linked the upper body with lower limbs across the pelvis in the back (Figure 1). The compression level was first determined for elastic and TPU fabrics separately, according to their relative stretch and then averaged over the whole suit. At specific 117 points, the compression level was even greater, for instance at the low waist and thigh lines (8.3 
and $6.7 \mathrm{mmHg}$, respectively). By determining the compression exactly and creating a

119 customized suit for each subject, identical apparel conditions were guaranteed for all subjects, and the possible effectiveness of the compressive suit was measurable. ${ }^{20}$ To determine to what extent wearing FBCS would influence trunk range of motion, trunk passive flexibility in frontal and sagittal planes was measured according to the literature. ${ }^{21}$ In addition, trunk rotation passive

flexibility was evaluated while participants lay on their backs with the right bent knee crossing over the thigh of the left extended leg. Participants remained relaxed and kept their back and both shoulders in contact with the floor, while the distance between the floor and knee was measured. Reduced mean trunk passive flexibility over all participants was obtained with FBCS compared to Control for flexion $(2.2 \mathrm{~cm})$ and rotation $(2 \mathrm{~cm})$, but was comparable for lateral flexion.

The two conditions were randomly assigned over all participants. According to pilot measurements, participants were given 30 minutes to become accustomed to the suit. During this period, standardized exercises were carried out based on runs, jumps and cuttings. When subjects started the measurement with FBCS, another 30 minutes practice was given to them prior to the Control condition.

Firstly, participants performed perturbed lateral reactive jumps (LRJ) to focus on the influence of FBCS on movement control during a functional task associated with high trunk lateral lean in the frontal plane. From a standing position, subjects performed a lateral reactive movement by jumping to their left side and then pushing off with their left leg to get back to their original position. Jump distance was set at $124 \%$ of the individual leg length. In order to induce lateral perturbations, subjects performed LRJ on a motor-driven plate, which could translate in the horizontal (medio-lateral) direction. The specially designed motor-driven plate (University of Sport Science, Freiburg, Germany) constituted of two independently moving plates $(1.15 \mathrm{~m} \times 0.3 \mathrm{~m}$ ), each driven by a separate motor (AKM51G, Danaher Motion, 
143 Düsseldorf, Germany). Details of this material have been previously described in the

144 literature. ${ }^{22}$ LRJ were performed, either without any perturbation (stable condition), or with a

145 perturbation to the left (sliding condition) or to the right (counteracting condition) as soon as

146 the foot made initial contact with the plate. Each perturbation consisted of a $10 \mathrm{~cm}$ translation

147 in either direction over $250 \mathrm{~ms}$, inducing a peak velocity of $0.55 \mathrm{~m} . \mathrm{s}^{-1}$ after $120 \mathrm{~ms}$.

148 Unpredictability was introduced using these three different landing modalities (stable, sliding,

149 counteracting) that were unknown prior to movement execution. Perturbation was triggered by

150 breaking a light beam (M18 series, Panasonic Electric Works Europe AG, Holzkirchen,

151 Germany), $0.04 \mathrm{~m}$ above the plate, to compensate for the delay in activation of the motor-driven

152 plate.

Secondly, participants were asked to perform either cutting maneuvers to $45^{\circ}$, crossovers to $-20^{\circ}$ or straight runs on a force plate (BP600900, AMTI®, Watertown, USA), after approaching the force plate (FP) at $4 \pm 0.2 \mathrm{~m} \cdot \mathrm{s}^{-1}$. The direction of the movement was indicated in a randomized order by a light signal occurring $650 \mathrm{~ms}$ before the left foot contacted the FP. The visual light stimulus was triggered automatically during the approach run via a light switch (M18 series, Panasonic Electric Works Europe AG, Holzkirchen, Germany) placed 3.7 $\mathrm{m}$ from FP. According to a light cue delay set individually, the $650 \mathrm{~ms}$ time could be achieved. The three lights were placed at eye level in the directions of the three tasks, $7 \mathrm{~m}$ away from the FP. The $45^{\circ}$ cutting maneuver to the right was the only movement to be further analyzed due to its relevance when investigating performance and injury aspects of changing direction tasks. Finally, three different performance tests were conducted to evaluate agility with the 5-

164 10-5 yards test, jump height, i.e. leg muscle power, with counter movement jumps (CMJ) and 165 core strength with a cable lift test. This latter test targets the action of hips, torso rotators, upper back, chest and shoulders while turning the trunk away from the strength machine as the hands first pull the rope cable handle towards the chest and then push up and away. The rationale for 
these tests is the importance of agility, leg muscle power and core strength for high performance during soccer, basketball or handball games. The good reliability of jump height measurements during CMJ has been reported in the literature (intraclass correlation coefficient $($ ICC $)=0.9),{ }^{23}$ as well as for the 5-10-5 yards agility test (ICC > 0.87). ${ }^{24}$ The time to accomplish the agility test was measured manually. Two CMJ were performed on the FP. The cable lift test was conducted twice at $70 \%$ of one-repetition maximum.

All participants were familiar with such movements due to their sport practice and were given enough practice trials to feel comfortable with all task modalities prior to the measurements.

Three-dimensional leg kinematics were recorded using reflective skin markers $(\varnothing 14$ $\mathrm{mm}$ ) placed on anatomical landmarks of the subjects' trunk (three markers), pelvis (four markers) and left thigh (three markers), shank (four markers) and foot (six markers). Markers were captured with a 12-camera motion analysis system (Vicon $\mathrm{MX}^{\mathrm{TM}}$, VICON Motion Systems Ltd.®, Oxford, UK) with a sampling frequency of $200 \mathrm{~Hz}$. A static trial with the subject standing in a pre-defined neutral position was used to define the kinematic model and to calculate segment lengths and joint centers. The kinematic modelling was performed with

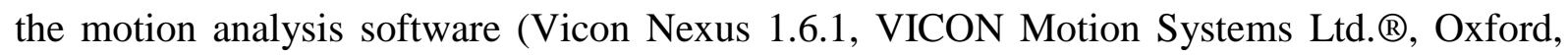
UK). Joint kinematics in three rotational degrees of freedom were determined using an yxz Euler rotation sequence of the respective segment coordinate system according to the global reference system defined in Vicon. This rotation sequence was around the flexion/extension axis first, then around the abduction/adduction axis followed by the rotation around the external/internal axis. This marker placement and kinematic modelling were used in previously published articles. ${ }^{7,19}$

Marker trajectory and ground reaction force signals were both filtered with a low pass Butterworth filter ( $4^{\text {th }}$ order, $15 \mathrm{~Hz}$ cut-off frequency) prior to calculating external joint 
193

194

195

196

197

198

199

200

201

202

203

204

205

206

207

208

209

210

211

212

213 214 of force and velocity.

215

216

217 external rotation.

moments with a standard inverse dynamics approach. Three-dimensional kinematic data of the trunk segment and hip joint were analyzed to understand the influence of FBCS on trunk positioning. Three-dimensional kinematics and kinetics of the knee joint were further analyzed to determine the influence of FBCS on knee joint stability and loading. Both kinematics and kinetics were measured during cutting maneuvers, but only kinematics was observed during LRJ. These variables were analyzed at the time of their peak values during the first half of ground contact during LRJ, to give some insights into how the apparel condition influences the range of motion of the different body segments. However, during cutting maneuvers, the different variables were determined at the time of peak knee abduction moment, as this is a relevant parameter associated with knee injury. ${ }^{25}$

Trunk lateral lean and trunk axial rotation were defined relative to the global coordinate system, i.e. deviations from the vertical axis and from the anterior-posterior axis, respectively. Both were positive for an orientation in the new movement direction (i.e. to the right direction). Hip rotation, knee rotation and knee rotation joint moment were defined as positive for an

The time to accomplish the 5-10-5 yards test served as outcome measure for agility. CMJ height was calculated based on the flight time method. During cable lift testing, the vertical acceleration signal of the accelerometer (University of Sport Science, Freiburg, Germany) placed on the top of the weight stack was filtered with a low pass Butterworth filter ( $4^{\text {th }}$ order, $20 \mathrm{~Hz}$ cut-off frequency) and then integrated to obtain velocity. Based on Newton's second law, lift force was calculated. Finally, power was determined by means of the product

The selected parameters were averaged across eight trials for the different LRJ modalities and the $45^{\circ}$ cutting maneuver, while the time during the 5-10-5 yards test was reported, and the highest $\mathrm{CMJ}$ and mean peak power value over two cable lift trials were 
218 calculated. These parameters served as the basis for the statistical analysis (Statistica 12,

219 StatSoft, Inc. ${ }^{\circledR}$, Tulsa, OK, US). All results are presented as group mean \pm standard deviation

220 (SD). After having confirmed that data followed a normal distribution (Shapiro-Wilk W),

221 sphericity was verified by means of the Mauchly's test. The influence of the apparel condition

222 on dependent variables for LRJ modalities was calculated using a two-way analysis of variance

223 (General Linear Model) with repeated measures to test for main effects of and interactions

224 between apparel (Control vs. FBCS) and LRJ modality (stable vs. sliding vs. counteracting). If

225 a statistically significant LRJ modality main effect was observed, Bonferroni corrected paired

226 comparisons determined where the differences occurred. The influence of the apparel condition

227 (Control vs. FBCS) on the dependent variables for the cutting maneuver and performance tasks

228 was analyzed using paired two-tailed t-tests. The magnitude of the changes was assessed with

229 effect size (ES) and 95\% confidence interval (CI). The ES was evaluated by means of partial 230 eta squared $\left(\eta^{2} \mathrm{p}\right)$ values and were considered small $(.01-.06)$, medium $(.06-.14)$ and large

231 (> .14) according to Cohen ${ }^{26}$. The level of significance was set at $P<.05$.

232

233

234

235

\section{Results}

There was a significant main effect of the apparel condition on hip rotation, knee abduction and rotation during LRJ (Table 1). Hip joint internal rotation was increased with FBCS $(P=.019)$, while knee joint abduction and internal rotation were reduced $(P=.002$ and $P<.001$, respectively). Statistical analysis revealed a significant main effect of the LRJ modality on trunk forward lean and trunk lateral lean $(P=.025$ and $P=.003$, respectively $)$, hip flexion, abduction and rotation $(P<.001$ for all), knee flexion and rotation (both $P<.001)$. However, no interaction effect (apparel condition x LRJ modality) was reported. Specifically, pairwise comparisons revealed that stable, sliding and counteracting modalities yielded significantly different values from each other for trunk lateral lean $(P<.004$ for all $)$, hip flexion $(P<.001$ for all $)$, hip rotation $(P<.003$ for all $)$, knee flexion $(P<.001$ for all $)$ and knee rotation 
$243(P<.003$ for all). Trunk flexion was significantly greater during counteracting than both stable

$244(P<.02)$ and sliding $(P<.001)$. Hip abduction was significantly greater during sliding than 245 both stable $(P<.001)$ and counteracting $(P<.001)$.

During the cutting maneuver, trunk forward lean and axial rotation were not influenced

247 by the apparel condition at the time of peak knee abduction moment, while trunk lateral lean 248 was significantly increased by FBCS $(P<.001)$. Hip joint internal rotation was higher for FBCS 249 than Control $(P=.003)$. Knee joint angles and moments, especially the knee abduction moment, were not influenced by the apparel condition (Table 2). The ensemble averages of trunk lateral lean and knee joint abduction moment over the stance phase of the cutting maneuver are presented (Figure 2).

Performance parameters were not significantly influenced by the apparel condition. The

sprint time measured during the 5-10-5 yards test was comparable between the two apparel 255 conditions $\left(5.6 \pm 0.3 \mathrm{~s}\right.$ for both conditions; $\left.P=.90,95 \% \mathrm{CI}[-0.15,0.13], \eta_{\mathrm{p}}^{2}=.002\right) . \mathrm{CMJ}$ height was not significantly different between Control and FBCS conditions $(0.28 \pm 0.05 \mathrm{~m}$ for both; $\left.P=.40,95 \% \mathrm{CI}[-0.01,0.01], \eta_{\mathrm{p}}^{2}=.07\right)$. Mean peak power produced by the participants during the cable lift did not differ significantly $\left(P=.55,95 \%\right.$ CI $\left.[-6,11], \eta_{\mathrm{p}}^{2}=.03\right)$ when performing the movement in the Control condition $(282 \pm 69 \mathrm{~W})$ or with FBCS $(280 \pm 68 \mathrm{~W})$.

\section{Discussion}

The main findings of the study were as follows: 1) FBCS did not reduce trunk angles during lateral movements, with even an increased trunk lateral lean during cutting maneuvers; 2) hip internal rotation was increased with FBCS; 3) knee joint moments were not reduced with FBCS; and 4) performance level was kept at the same level.

Perturbed lateral reactive jumps yielded significantly different segment and joint 
modality at the time of initial contact has already been provided in the literature. ${ }^{27}$ But during

269 perturbed lateral reactive jumps, trunk positioning was not influenced by the compression 270 garment. It seems that despite a suit design enabling a reduced passive trunk range of motion, 271 FBCS cannot support the trunk segment during an active dynamic movement. Thus, the trunk 272 segment was not supported either during cutting maneuvers. On the contrary, trunk lateral lean 273 was even significantly increased by $4^{\circ}$ while wearing FBCS during $45^{\circ}$ cutting maneuvers.

274 While all participants showed greater trunk lateral lean with FBCS than without apparel, five 275 subjects reached particularly large values around $15^{\circ}$ with FBCS. Although literature 276 demonstrated that compression garments could reduce the active range of motion of the hip 277 joint during a non-athletic task ${ }^{13}$ and during sprinting, ${ }^{14}$ a more neutral trunk positioning 278 through apparel could not be achieved during unanticipated lateral movements. It might be 279 possible that participants would rely on the support of the suit according to the compression and elasticity properties felt by the participants, thereby reducing their active trunk muscle control. But the lack of apparel support would bring participants to this higher trunk lateral lean in the frontal plane during cutting maneuvers.

The specific FBCS linked shoulders with knees across the pelvis in the back thanks to the elastic straps design. But at the hip joint level, only hip internal rotation was altered. The hip internal rotation observed in the present study is consistent with literature during lateral reactive jumps ${ }^{27}$ and cutting manoeuvres. ${ }^{28}$ However, increased peak internal rotation values with FBCS might rather reflect a lack of strength in the external hip rotators in female athletes, ${ }^{29}$ exacerbated by 288 the additional tension of the compression garment.

It appears that external passive support with the aim of enhancing trunk positioning during dynamic lateral movements classically encountered during team sports is difficult to achieve with apparel. At this point, further improvements to the design of the compression suit does not seem possible as several years of research and development were needed to yield this 
current comfortable full-body suit, which enables reduced passive trunk flexibility through the combined compression and elasticity properties. In addition, garments were customized to each participant in the present study. Further increasing the compression level of the suit to further support body segments, i.e. by reaching typical values of compression socks $>15 \mathrm{mmHg},{ }^{30}$ does not seem possible given that i) the straps used already increase the stiffness of the suit and ii) the higher compression level would reduce the comfort level necessary to perform wholebody dynamic movements.

Trunk and hip control alterations with FBCS could have consequences at the knee joint level. Indeed, higher trunk lateral lean during cutting maneuvers while wearing FBCS might be associated with increased knee joint loading. ${ }^{6,7}$ In present study, higher trunk lateral lean had no deleterious influence on the knee joint, as no significant difference in knee abduction moment was evident between FBCS and control conditions. Increased peak internal hip rotation angles with FBCS might reflect a lack of strength in the external hip rotators ${ }^{29}$ and further a critical knee joint position (position of no return), where the hip moves more rapidly into adduction and internal rotation and the knee collapses in valgus. ${ }^{31}$ But the consequences at the knee joint level during lateral reactive jumps were rather a reduction of the abduction and internal rotation angles whatever the landing condition with FBCS which speaks in favor of better knee joint alignment. Thus, alteration of trunk positioning during lateral movements with FBCS was not associated with changes in knee joint stability.

This result does not support the theoretical model proposed by Hewett and $\mathrm{Myer}^{3}$ and is not in agreement with experimental results, where increased trunk lateral lean was associated with increased knee joint abduction moment. ${ }^{6,7}$ This finding underlines the fact that trunk positioning only partially influences knee joint loading, and that relatively small trunk angle variations $\left(4^{\circ}\right)$, albeit significant, may not always have an effect on knee abduction moment, especially when considering the large variability of this knee joint loading parameter. 
It is worth noticing that FBCS had no influence on any of the performance parameters.

319 The same level of agility while wearing the compression suit or in the control condition during the 5-10-5 yards test is consistent with the literature. ${ }^{13,16,32}$ Although improvement of jumping performance with a compression garment has been reported in the literature, ${ }^{14,33}$ it is interesting to note that jump height was not impaired with the compression suit targeting trunk and pelvis positioning rather than the lower limbs in the present study. Finally, core strength during the cable lift task was kept at the same level when performed with FBCS. Accordingly, FBCS can be used by athletes without risk of compromising performance.

This study has some limitations that need to be considered. The moderate reliability of knee kinetics during dynamic tasks such as cutting tasks would suggest the need for a greater sample size than 12 participants. ${ }^{34}$ In the present study, measured variables were assessed over eight trials to ensure representative values for each participant. But the inter-subject variability was pretty high for joint kinetics and the associated statistical power low $(<0.2)$. Thus, future studies would possibly need a larger sample size to tease out effects on knee joint kinetics or at least enable to discuss the lack of difference in knee joint loading. Another potential limitation was the use of a cable lift task. While this test seems to be relevant to evaluate core strength, 334 the validity and reliability of this measure would need to be assessed to determine its measurement error. This will allow evaluating the importance of observed differences from a statistical perspective as well as in terms of its practical implication. body compression suit, trunk kinematics could not be favorably influenced. Achieving external 339 support of trunk positioning in female athletes through apparel seems to be difficult during unanticipated lateral movements. However, a full body compression suit did not impair any of the performance parameters and even yielded a better alignment of the knee during lateral reactive jumps. 
345 1. Olsen O-E, Myklebust G, Engebretsen L, Bahr R. Injury mechanisms for anterior cruciate ligament injuries in team handball: a systematic video analysis. Am J Sports Med. 2004;32(4):1002-1012.

2. Alentorn-Geli E, Myer GD, Silvers HJ, et al. Prevention of non-contact anterior cruciate ligament injuries in soccer players. Part 1: Mechanisms of injury and underlying risk factors. Knee Surg Sports Traumatol Arthrosc. 2009;17(7):705-729. doi:

3. Hewett TE, Myer GD. The mechanistic connection between the trunk, hip, knee, and anterior cruciate ligament injury. Exerc Sport Sci Rev. 2011;39(4):161-166. doi:10.1097/JES.0b013e3182297439

4. Hughes G. A review of recent perspectives on biomechanical risk factors associated with 356 anterior cruciate ligament injury. Res Sports Med. 2014;22(2):193-212. doi:10.1080/15438627.2014.881821

5. Hewett TE, Torg JS, Boden BP. Video analysis of trunk and knee motion during non-contact anterior cruciate ligament injury in female athletes: lateral trunk and knee abduction motion are combined components of the injury mechanism. Br J Sports Med. 2009;43(6):417-422. doi:10.1136/bjsm.2009.059162

6. Jamison ST, Pan X, Chaudhari AMW. Knee moments during run-to-cut maneuvers are associated with lateral trunk positioning. J Biomech. 2012;45(11):1881-1885. doi:10.1016/j.jbiomech.2012.05.031

365 7. Weltin E, Mornieux G, Gollhofer A. Influence of gender on trunk and lower limb 366 biomechanics during lateral movements. Res Sports Med. 2015;23(3):265-277. doi:10.1080/15438627.2015.1040915

8. Dempsey AR, Lloyd DG, Elliott BC, Steele JR, Munro BJ, Russo KA. The effect of 
technique change on knee loads during sidestep cutting. Med Sci Sports Exerc. 2007;39(10):1765-1773.

9. Frank B, Bell DR, Norcross MF, Blackburn JT, Goerger BM, Padua DA. Trunk and hip biomechanics influence anterior cruciate loading mechanisms in physically active participants. Am $\quad J \quad$ Sports

Med. 2013;41(11):2676-2683.

375 376 doi:10.1177/0363546513496625

10. Agel J, Arendt EA, Bershadsky B. Anterior cruciate ligament injury in national collegiate athletic association basketball and soccer: a 13-year review. Am J Sports Med. 2005;33(4):524-530. doi:doi:10.1177/0363546504269937

11. Renstrom P, Ljungqvist A, Arendt E, et al. Non-contact ACL injuries in female athletes: an International Olympic Committee current concepts statement. Br J Sports Med. 2008;42(6):394-412. doi:10.1136/bjsm.2008.048934

12. MacRae BA, Cotter JD, Laing RM. Compression garments and exercise. Garment considerations, physiology and performance. Sports Med. 2011;41(10):815-843. doi:10.2165/11591420-000000000-00000

13. Bernhardt T, Anderson GS. Influence of moderate prophylactic compression on sport performance. J Strength Cond Res. 2005;19(2):292-297.

14. Doan BK, Kwon Y-H, Newton RU, et al. Evaluation of a lower-body compression garment. J Sports Sci. 2003;21(8):601-610. doi:10.1080/0264041031000101971

15. Chaudhari AMW, Jamison ST, McNally MP, Pan X, Schmitt LC. Hip adductor activations during run-to-cut manoeuvres in compression shorts: implications for return to sport after groin injury. $J$ Sports Sci. 2014;32(14):1333-1340. doi:10.1080/02640414.2014.889849

16. Duffield R, Portus M. Comparison of three types of full-body compression garments on throwing and repeat-sprint performance in cricket players. $\mathrm{Br} J$ Sports Med. 

2007;41(7):409-414. doi:10.1136/bjsm.2006.033753

395 17. Sperlich B, Haegele M, Achtzehn S, Linville J, Holmberg H-C, Mester J. Different types of 396 compression clothing do not increase sub-maximal and maximal endurance performance in well-trained athletes. $J$ Sports Sci. 2010;28(6):609-614. 398 doi:10.1080/02640410903582768

399

400

401

402

403

404

405

406

407

408

409

410

411

412

413

414

415

416

417

418

18. Sear JA, Hoare TK, Scanlan AT, Abt GA, Dascombe BJ. The effects of whole-body compression garments on prolonged high-intensity intermittent exercise. J Strength Cond Res. 2010;24(7):1901-1910. doi:10.1519/JSC.0b013e3181db251b

19. Mornieux G, Gehring D, Fürst P, Gollhofer A. Anticipatory postural adjustments during cutting manoeuvres in football and their consequences for knee injury risk. J Sports Sci. 2014;32(13):1255-1262. doi:10.1080/02640414.2013.876508

20. Troynikov O, Ashayeri E, Burton M, Subic M, Alam F, Marteau S. Factors influencing the effectiveness of compression garments used in sports. Procedia Eng. 2010;2(2):28232829. doi:10.1016/j.proeng.2010.04.073

21. Yoshida A, Kahanov L. The effect of kinesio taping on lower trunk range of motions. Res Sports Med. 2007;15(2):103-112. doi:10.1080/15438620701405206

22. Mornieux G, Gehring D, Tokuno C, Gollhofer A, Taube W. Changes in leg kinematics in response to unpredictability in lateral jump execution. Eur J Sport Sci. 2014;14(7):678685. doi:10.1080/17461391.2014.894577

23. Moir G, Shastri P, Connaboy C. Intersession reliability of vertical jump height in women and men. J Strength Cond Res. 2008;22(6):1779-1784. doi:10.1519/JSC.0b013e318185f0df

24. Stewart PF, Turner AN, Miller SC. Reliability, factorial validity, and interrelationships of five commonly used change of direction speed tests. Scand J Med Sci Sport. 2014;24(3):500-506. doi:10.1111/sms.12019 
25. Hewett TE, Myer GD, Ford KR, et al. Biomechanical measures of neuromuscular control and valgus loading of the knee predict anterior cruciate ligament injury risk in female athletes: a prospective study. Am J Sports Med. 2005;33(4):492-501.

26. Cohen J. Statistical power analysis for the behavorial sciences. $2^{\text {nd }}$ ed. Hillsdale, New Jersey: Lawrence Erlbaum Associates, Publishers; 1988.

27. Weltin E, Gollhofer A, Mornieux G. Effect of gender on trunk and pelvis control during lateral movements with perturbed landing. Eur J Sport Sci. 2016;16(2):182-189. doi:10.1080/17461391.2014.992478

28. Beaulieu ML, Lamontagne $\mathrm{M}, \mathrm{Xu}$ L. Lower limb muscle activity and kinematics of an unanticipated cutting manoeuvre: a gender comparison. Knee Surg Sports Traumatol Arthrosc. 2009;17(8):968-976. doi:10.1007/s00167-009-0821-1

29. Leetun DT, Ireland ML, Willson JD, Ballantyne BT, Davis IM. Core stability measures as risk factors for lower extremity injury in athletes. Med Sci Sports Exerc. 2004;36(6):926-934.

30. Ali A, Creasy RH, Edge JA. Physiological effects of wearing graduated compression stockings during running. Eur J Appl Physiol. 2010;109(6):1017-1025. doi:10.1007/s00421-010-1447-1

31. Ireland ML. Anterior cruciate ligament injury in female athletes: epidemiology. $J$ Athl Train. 1999;34(2):150-154.

32. Davies V, Thompson KG, Cooper S. The effects of compression garments on recovery. $J$ Strength Cond Res. 2009;23(6):1786-1794. doi:10.1519/JSC.0b013e3181b42589

33. Kraemer WJ, Bush JA, Bauer JA, et al. Influence of compression garments on vertical jump performance in NCAA Division I volleyball players. J Strength Cond Res. 1996;10(3):180-183.

34. Sankey SP, Raja Azidin RM, Robinson MA, et al. How reliable are knee kinematics and 
kinetics during side-cutting manoeuvres? Gait Posture. 2015;41(4):905-911.

445 doi:10.1016/j.gaitpost.2015.03.014

446 


\section{Tables}

Table 1 Mean \pm SD values of the different kinematic variables during lateral reactive jumps (LRJ) performed in Control and full body compression suit (FBCS) conditions.

\begin{tabular}{|c|c|c|c|c|c|}
\hline Variable & Control (pooled LRJ) & FBCS (pooled LRJ) & $P$ value & $95 \% \mathrm{CI}$ & $\operatorname{ES}\left(\eta_{p}^{2}\right)$ \\
\hline Trunk Forward Lean $\left(^{\circ}\right)$ & $32.0 \pm 6.2$ & $31.6 \pm 6.8$ & .66 & {$[-1.7,2.5]$} & .02 \\
\hline Trunk Lateral Lean $\left(^{\circ}\right)$ & $-7.5 \pm 5.4$ & $-7.9 \pm 6.1$ & .63 & {$[-1.4,2.2]$} & .02 \\
\hline Trunk Right Axial Rotation $\left(^{\circ}\right)$ & $4.6 \pm 9.4$ & $6.7 \pm 9.3$ & .10 & {$[-4.7,0.5]$} & .23 \\
\hline Hip Flexion $\left(^{\circ}\right)$ & $50.6 \pm 8.9$ & $51.1 \pm 13.3$ & .84 & {$[-5.5,4.5]$} & $<.01$ \\
\hline Hip Abduction $\left(^{\circ}\right)$ & $35.5 \pm 4.7$ & $35.7 \pm 5.7$ & .84 & {$[-2.5,2.0]$} & $<.01$ \\
\hline Hip Ext. Rotation $\left(^{\circ}\right)$ & $-5.0 \pm 6.5$ & $-11.1 \pm 6.2^{*}$ & .019 & {$[1.2,10.9]$} & .41 \\
\hline Knee Flexion $\left(^{\circ}\right)$ & $59.0 \pm 5.3$ & $59.9 \pm 5.1$ & .10 & {$[-2.0,0.2]$} & .23 \\
\hline Knee Abduction $\left(^{\circ}\right)$ & $10.3 \pm 6.7$ & $5.3 \pm 3.9 * *$ & .002 & {$[2.3,7.5]$} & .61 \\
\hline Knee Ext. Rotation $\left(^{\circ}\right)$ & $-13.5 \pm 7.4$ & $-8.6 \pm 6.5 * * *$ & $<.001$ & {$[-7.3,-2.6]$} & .66 \\
\hline
\end{tabular}

The influence of the apparel condition is reported using $P, 95 \%$ confidence interval $(95 \% \mathrm{CI})$ and effect size (ES) with partial eta squared $\left(\eta^{2} \mathrm{p}\right)$ values.

${ }^{*}, * *, * * *$ indicates significant differences between Control and FBCS with $P<.05, P<.01$ and $P<.001$, respectively. 
Table 2 Mean \pm SD values of the different kinematic and joint moment variables during cutting maneuvers performed in Control and full body compression suit (FBCS) conditions.

\begin{tabular}{|c|c|c|c|c|c|}
\hline Variable & Control & FBCS & $P$ value & $95 \% \mathrm{CI}$ & $\operatorname{ES}\left(\eta_{p}^{2}\right)$ \\
\hline Trunk Forward Lean $\left(^{\circ}\right)$ & $12.6 \pm 7.8$ & $13.9 \pm 5.6$ & .22 & {$[-3.6,0.9]$} & .14 \\
\hline Trunk Lateral Lean $\left(^{\circ}\right)$ & $-5.1 \pm 3.7$ & $-8.7 \pm 5.0 * * *$ & $<.001$ & {$[2.2,5.0]$} & .74 \\
\hline Trunk Right Axial Rotation $\left(^{\circ}\right)$ & $-8.5 \pm 8.4$ & $-7.8 \pm 7.5$ & .61 & {$[-3.4,2.1]$} & .02 \\
\hline Hip Flexion $\left(^{\circ}\right)$ & $51.6 \pm 6.7$ & $49.3 \pm 10.6$ & .35 & {$[-2.9,7.5]$} & .08 \\
\hline Hip Abduction $\left(^{\circ}\right)$ & $8.4 \pm 8.9$ & $7.7 \pm 5.9$ & .59 & {$[-2.2,3.7]$} & .03 \\
\hline Hip Ext. Rotation $\left(^{\circ}\right)$ & $-4.2 \pm 7.3$ & $-9.8 \pm 4.4 * *$ & .003 & {$[2.4,8.8]$} & .57 \\
\hline Knee Flexion $\left(^{\circ}\right)$ & $28.3 \pm 5.0$ & $28.0 \pm 6.1$ & .56 & {$[-0.9,1.6]$} & .03 \\
\hline Knee Abduction $\left(^{\circ}\right)$ & $4.7 \pm 3.1$ & $2.7 \pm 3.1$ & .054 & {$[-0.1,4.1]$} & .30 \\
\hline Knee Ext. Rotation $\left(^{\circ}\right)$ & $-7.4 \pm 6.6$ & $-3.4 \pm 5.2$ & .052 & {$[-8.1,0.1]$} & .30 \\
\hline Knee Flexion Moment $\left(\mathrm{Nm} \cdot \mathrm{kg}^{-1}\right)$ & $0.16 \pm 0.65$ & $0.20 \pm 0.75$ & .74 & {$[-0.33,0.24]$} & .01 \\
\hline Knee Abduction Moment (Nm. $\left.\mathrm{kg}^{-1}\right)$ & $1.31 \pm 0.46$ & $1.20 \pm 0.46$ & .30 & {$[-0.12,0.35]$} & .1 \\
\hline Knee Ext. Rotation Moment $\left(\mathrm{Nm} \cdot \mathrm{kg}^{-1}\right)$ & $-0.13 \pm 0.11$ & $-0.16 \pm 0.12$ & .42 & {$[-0.06,0.13]$} & .06 \\
\hline
\end{tabular}

The influence of the apparel condition is reported using $P, 95 \%$ confidence interval $(95 \% \mathrm{CI})$ and effect size $(\mathrm{ES})$ with partial eta squared $\left(\eta^{2} \mathrm{p}\right)$ values.

**,*** indicates significant differences between Control and FBCS with $P<.01$ and $P<.001$, respectively. 


\section{Figure Captions}

Figure 1 - Participant wearing the customized full body compression suit (FBCS) with 2x thermoplastic polyurethane layers (TPU). The FBCS design provides support for the trunk over the pelvis segment through the 2x TPU layers that link the upper body with lower limbs across the pelvis in the back (right). The customized apparel production ensures a perfect fitting for each participant.

Figure 2 - Ensemble averages of lateral trunk flexion in the frontal plane and knee joint abduction moment for Control (black line) and full body compression suit (FBCS, grey line) during the foot contact phase of the cutting maneuver. 